\title{
A CHARACTERISATION OF CLOSED SUBALGEBRAS OF $\mathscr{B}(H)$
}

\author{
P. G. DIXON
}

(Received 30th April 1975)

We show that the class of Banach algebras $A$ isomorphic with normclosed (non-self-adjoint) subalgebras of $\mathscr{B}(H)$ is characterized by the condition that the norms of polynomials in $A$ be dominated by the norms of the same polynomials in $\mathscr{B}(H)$.

Definition 1. If $H$ is a Hilbert space, $\mathscr{B}(H)$ denotes the Banach algebra of all bounded operators on $H$. A Banach algebra which is bicontinuously isomorphic with a closed subalgebra of $\mathscr{B}(H)$, for some Hilbert space $H$, will be called an $R$-algebra, and an $I R$-algebra if the isomorphism is isometric. If $X$ is a compact Hausdorff space, then $C(X)$ denotes the Banach algebra of all continuous complex-valued functions on $X$, with the sup norm. A uniform algebra is a closed subalgebra of some $C(X)$. A Q-algebra is a Banach algebra $A$ which is bicontinuously isomorphic with the quotient of a uniform algebra by a closed ideal, and $A$ is an IQ-algebra if the isomorphism is isometric.

Definition 2. Unless otherwise qualified, the word "polynomial" will mean a polynomial in several non-commuting variables, without constant term. If $p\left(X_{1}, \ldots, X_{n}\right)$ is such a polynomial, $A$ a Banach algebra and $\delta>0$, then we define

$$
\|p\|_{A, \delta}=\sup \left\{\left\|p\left(x_{1}, \ldots, x_{n}\right)\right\|: x_{i} \in A,\left\|x_{i}\right\| \leqslant \delta(1 \leqslant i \leqslant n)\right\} .
$$

We have separate notations for two important special cases: $\|p\|_{\infty}$ for $\|p\|_{C, 1}$, where $C$ denotes the complex numbers, and $\|p\|_{\eta}$ for $\|p\|_{\mathscr{B}(H), 1}$, where $H$ is, say, a separable Hilbert space; (any other infinite-dimensional Hilbert space $H$ produces the same norm).

We recall the main results about $Q$-algebras and their relation to R-algebras.

Theorem (Craw; see (2)). A commutative Banach algebra $A$ is a $Q$-algebra if and only if there exist $M, \delta>0$ such that $\|p\|_{A, \delta} \leqslant M\|p\|_{\infty}$ for all polynomials $p$. Further, $A$ is $I Q$ if and only if this condition holds with $M=\delta=1$.

Theorem (Cole; see (2)). Every Q-algebra is an R-algebra.

Theorem (Varopoulos (4)). Not every commutative $R$-algebra is $Q$ algebra. 
Our theorem is a sort of non-commutative analogue of Craw's result, though, by Varopoulos' theorem, it reduces to something different in the commutative case (see Corollary).

Theorem. A Banach algebra $A$ is an $R$-algebra if and only if there exist $M, \delta>0$ such that $\|p\|_{A, \delta} \leqslant M\|p\|_{\eta}$ for all polynomials $p$. Further, $A$ is IR if and only if this condition holds with $M=\delta=1$.

Proof. That every R-algebra satisfies the stated condition, with $M=$ $\delta=1$ for an IR-algebra, is clear. The proof of the converse parallels that of Craw's theorem, using $\mathscr{B}(H)$ instead of $C$. Thus, we let $\Lambda=$ $\{a \in A:\|a\| \leqslant \delta\}, \Delta=\{z \in \mathscr{B}(H): \| z \mid \leqslant 1\}$, and $X$ the Cartesian product $\Delta^{\Lambda}$. Let $B(X, \mathscr{B}(H))$ denote the $C^{*}$-algebra of all bounded functions $\phi: X \rightarrow$ $\mathscr{B}(H)$, with the sup norm: $\|\phi\|=\sup \{\|\phi(x)\|: x \in X\}$. For each $a \in \Lambda$, we define $\zeta_{a} \in B(X, \mathscr{B}(H))$ by $\zeta_{a}(x)=x(a)(x \in X)$. Let $U_{0}$ be the subalgebra of $B(X, B(H))$ generated by $\left\{\zeta_{a}: a \in \Lambda\right\}$, and let $U$ be the closure of $U_{0}$. Let $\pi$ be the homomorphism of $U_{0}$ onto $A$ defined by

$$
\pi\left(p\left(\zeta_{a_{1}}, \ldots, \zeta_{a_{n}}\right)\right)=p\left(a_{1}, \ldots, a_{n}\right)
$$

for all polynomials $p\left(X_{1}, \ldots, X_{n}\right)$ and all $n$-tuples $\left(a_{1}, \ldots, a_{n}\right)$ of distinct elements of $\Lambda$. Since $\left\|\zeta_{a}\right\|=1(a \in \Lambda)$, the given condition $\|p\|_{A, \delta} \leqslant M\|p\|_{\eta}$ ensures that $\pi$ is continuous, with norm at most $M$. Therefore $\pi$ extends to a homomorphism of $U$ onto $A$. Thus $A$ is bicontinuously isomorphic with a quotient of the closed subalgebra of $U$ of the $C^{*}$-algebra $B(X, \mathscr{B}(H)$ ).

The remainder of the proof is a non-commutative analogue of Cole's theorem, due to Bernard.

Theorem (Bernard (1)). Let $\Gamma$ be a $C^{*}$-algebra with identity. Let $U$ be a closed subalgebra of $\Gamma$ containing the identity, and let $I$ be a closed ideal of $U$. Then $U / I$ is an IR-algebra.

The provisos concerning the identity may clearly be dropped, by adjoining the identity to $U$ if it is not already in $U$. Applying Bernard's theorem to our situation shows that $A$ is an R-algebra.

If $M=\delta=1$, then the isomorphism induced by $\pi$ is an isometry, and the isometric nature of Bernard's theorem completes the proof that $A$ is IR.

For the commutative version, we define $\|p\|_{\eta}$ for a polynomial $p\left(X_{1}, \ldots, X_{n}\right)$ in commuting variables by

$$
\|p\|_{\eta}=\sup \left\|p\left(T_{1}, \ldots, T_{n}\right)\right\|
$$

the supremum being taken over all $n$-tuples $\left(T_{1}, \ldots, T_{n}\right)$ of commuting contractions on a separable Hilbert space. 
Corollary 1. A commutative Banach algebra $A$ is an $R$-algebra if and only if there exist $M, \delta>0$ such that $\|p\|_{A, \delta} \leqslant\|p\|_{\eta}$ for all polynomials $p\left(X_{1}, \ldots, X_{n}\right)$ in commuting variables $X_{1}, \ldots, X_{n}$ and without constant term. Further, commutative IR-algebras are characterised by this condition with $M=\delta=1$.

The main force of the theorem is that there is some condition on the norms of polynomials which characterises R-algebras. Of course, the function $\|\cdot\|_{\eta}$ is not easily calculated, and there is a need for more usable conditions. However, the fact that there is a condition of this form is of some help. By methods similar to those used by Davie ((3) pp. 38-39) to construct Arens regular, non- $Q$ algebras, we may prove:

Corollary 2. There exist commutative, Arens regular, non- $R$ algebras.

\section{REFERENCES}

(1) A. BERNARD, Quotients of operator algebras, Seminar on uniform algebras (University of Aberdeen, 1973).

(2) F. F. BONSALl and J. DUNCAN, Complete normed algebras (Springer, Berlin, 1973).

(3) A. M. DAvIE, Quotient algebras of uniform algebras, J. London Math. Soc. (2), 7 (1973), 31-40.

(4) N. Th. Varopoulos, Sur une inégalité de von Neumann, C.R. Acad. Sci. Paris 277 (1973), 19-22.

Department of Pure Mathematics

THE UNIVERSITY

SHEFFIELD, S3 7RH

ENGLAND 